\title{
Health-related quality of life and utility outcomes with selinexor in relapsed/refractory diffuse large B-cell lymphoma
}

Jatin Shah1, Sharon Shacham¹, Michael Kauffman1, Patrick Daniele*,2, Dimitrios Tomaras²,

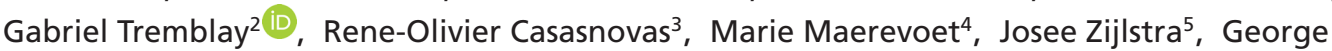
Follows $^{6}$, Joost S. P Vermaat ${ }^{7}$, Nagesh Kalakonda ${ }^{8}$, Andre Henri Goy ${ }^{9}$, Sylvain Choquet $^{10}$, Eric Van Den Neste ${ }^{11}$, Brian T Hill ${ }^{12}$, Catherine Thieblemont ${ }^{13}$, Federica Cavallo ${ }^{14}$, Fátima de la Cruz ${ }^{15}$, John Kuruvilla ${ }^{16}$, Nada Hamad ${ }^{17}$, Reda Bouabdallah ${ }^{18}$, Ulrich Jäger ${ }^{19}$, Paolo Caimi $^{20}$, Ronit Gurion ${ }^{21}$, Krzysztof Warzocha ${ }^{22}$, Sameer Bakhshi' ${ }^{23}$, Juan Manuel Sancho ${ }^{24}$, Michael Schuster ${ }^{25}$, Miklós Egyed ${ }^{26}$, Fritz Offner ${ }^{27}$, Theodoros P Vasilakopoulos ${ }^{28}$, Priyanka Samal $^{29}$, Agnes Nagy ${ }^{30}$, Matthew $\mathrm{Ku}^{31}$ \& Miguel Ángel Canales Albendea ${ }^{32}$

${ }^{1}$ Karyopharm Therapeutics, Inc., Newton, MA 02459, USA

${ }^{2}$ Health Economics, Purple Squirrel Economics, Montreal, QC H3J 1M1, Canada

${ }^{3}$ Clinical Hematology, CHU Dijon Bourgogne, Dijon, 21079, France

${ }^{4}$ Hématologie, Institut Jules Bordet, Brussels, 1000, Belgium

${ }^{5}$ Department of Hematology, Amsterdam UMC, Amsterdam, 1105 AZ, The Netherlands

${ }^{6}$ Haematology, Addenbrooke's Hospital, Cambridge, CB2 OQQ, UK

${ }^{7}$ Hematology, Leiden University Medical Center, Leiden, 2333 ZA, The Netherlands

${ }^{8}$ Institute of Translational Medicine, University of Liverpool, Liverpool, L3 9TA, UK

${ }^{9}$ John Theurer Cancer Center, Hackensack University Medical Center, Hackensack, NJ 07601, USA

${ }^{10}$ Service d'Hématologie clinique, Hôpital Pitié-Salpêtrière, Paris, 75013, France

${ }^{11}$ Faculté de médecine et médecine dentaire, Université catholique de Louvain, Ottignies-Louvain-la-Neuve, 1348, Belgium

${ }^{12}$ Hematology \& Medical Oncology, Cleveland Clinic, Cleveland, OH 44106, USA

${ }^{13}$ Hémato-Oncologie, Hôpital Saint-Louis, Paris, 75010, France

${ }^{14}$ Divisione di Ematologia, University of Turin, Turin, 10138, Italy

${ }^{15}$ Facultativo Especialista de Área de Hematología, Hospital Universitario Virgen del Rocío, Sevilla, 41013, Spain

${ }^{16}$ Cancer Clinical Research Unit, University Health Network Research, Toronto, ON, Canada

${ }^{17}$ Haematology Clinical Trials Unit, St Vincent's Hospital, Melbourne, 3065, Australia

${ }^{18}$ Hematology, Institut Paoli-Calmettes, Marseille, 13009, France

${ }^{19}$ Department of Clinical Hematology \& Hemostaseology, Medical University of Vienna, Vienna, 1090, Austria

${ }^{20}$ Department of Medicine, UH Cleveland Medical Center, Cleveland, OH 44106, USA

${ }^{21}$ Hematology, Tel Aviv University, Tel Aviv, 69978, Israel

${ }^{22}$ Institute of Hematology \& Blood Transfusion, Warsaw, 02-776, Poland

${ }^{23}$ Department of Medical Oncology, Institute Rotary Cancer Hospital, New Delhi, 110029, India

${ }^{24}$ Department of Clinical Hematology, Germans Trias i Pujol Hospital, Barcelona, 08916, Spain

${ }^{25}$ Hematology, Stony Brook University, NY 11794, USA

${ }^{26}$ Department of Haematology, Somogy County Kaposi Mór Hospital, Kaposvár, 7400, Hungary

${ }^{27}$ Department of Clinical Hematology, Ghent University Hospital, Ghent, 9000, Belgium

${ }^{28}$ Faculty of Medicine, National \& Kapodistrian University of Athens, Athens, 10679, Greece

${ }^{29}$ IMS \& SUM Hospital, Bhubaneswar, Odisha, India

${ }^{30}$ Hematology, Semmelweis University, Budapest, 1085, Hungary

${ }^{31}$ Department of Haematology, St Vincent's Hospital, Melbourne, VIC, 3065, Australia

${ }^{32}$ Servicio de Hematología, Hospital Universitario La Paz, Madrid, 28046, Spain

*Author for correspondence: patrickdaniele@pshta.com

Aim: Evaluate health-related quality of life (HRQoL) and health utility impact of single-agent selinexor in heavily pretreated patients with relapsed/refractory diffuse large B-cell lymphoma. Patients \& methods: Functional Assessment of Cancer Therapy (FACT) - Lymphoma and EuroQoL five-dimensions fivelevels data collected in the single-arm Phase Ilb trial SADAL (NCT02227251) were analyzed with mixedeffects models. Results: Treatment responders maintained higher FACT - Lymphoma ( $p \leq 0.05)$, FACT General ( $p<0.05$ ) and EuroQoL five-dimensions five-levels index scores $(p<0.001)$ beginning in cycle 3 . The estimated difference in health state utilities for treatment response and progressive disease was both statistically significant and clinically meaningful (mean difference: $0.07 ; p=0.001)$. Conclusion: In patients 
with relapsed/refractory diffuse large B-cell lymphoma, objective response to selinexor was associated with HRQoL maintenance, reduction in disease-related HRQoL decrements and higher health utilities.

Lay abstract: This work examined quality of life (QoL) among patients with relapsed/refractory diffuse large B-cell lymphoma with two to five prior therapies who received single-agent selinexor in the SADAL clinical trial. Analysis of patient-reported Functional Assessment of Cancer Therapy - Lymphoma and EuroQoL five-dimensions five-levels data showed that patients who had objective clinical response to selinexor maintained their QoL over the course of treatment. Grade $\geq 3$ adverse events and serious adverse events were not associated with clinically meaningful negative QoL impacts.

Clinical trial registration: NCT02227251 (ClinicalTrials.gov)

First draft submitted: 14 September 2020; Accepted for publication: 3 December 2020; Published online: 2 February 2021

Keywords: diffuse large B-cell lymphoma • disutility of adverse events $\bullet$ EQ-5D-5L • FACT-Lym • health-related quality of life $\bullet$ health state utility $\bullet$ health utility $\bullet$ patient-reported outcomes $\bullet$ selinexor

Diffuse large B-cell lymphoma (DLBCL) is an aggressive, fast-growing malignancy of B-lymphocytes [1,2]. DLBCL is the most common subtype of non-Hodgkin lymphoma (NHL) malignancies and accounted for $25 \%$ of NHL cases in 2016 in the USA [3]. According to the Surveillance, Epidemiology, and End Results Program data from 2012 to 2016, the incidence of DLBCL in the USA was 5.6 per 100,000 per year [4]. Despite high cure rates with first-line standard of care chemoimmunotherapy regimens, an estimated $30-40 \%$ of patients relapse [1,5-10]. In the second-line setting, fit, transplant-eligible patients may progress to high-dose therapy and autologous stem cell transplantation (ASCT), leading to long-term remission for some patients. However, for patients who relapse after salvage therapy, with or without ASCT, outcomes are especially poor, with median overall survival (OS) in cohort studies ranging from 4.7 to 6.6 months from commencement of salvage therapy [11-17]. While no standard of care treatment regimen exists in the third-line setting, options include emergent therapies such as chimeric antigen receptor (CAR) T-cell therapy and polatuzumab vedotin plus rituximab and bendamustine, as well as alternate chemoimmunotherapy regimens, clinical trials, palliative radiation therapy or best supportive care [5].

Selinexor is an orally bioavailable selective nuclear export inhibitor [18-21]. Selinexor was approved in 2020 for the treatment of adult patients with relapsed/refractory DLBCL (RR DLBCL), not otherwise specified, after two prior lines of systemic therapy [22]. Safety and efficacy of selinexor were demonstrated in the single-arm, Phase IIb, open-label, multicenter SADAL trial in transplant-ineligible patients with RR DLBCL who received two to five prior systemic therapies ( $\mathrm{n}=127$ in the modified intent-to-treat [mITT] population) [22,23]. The SADAL trial met the primary end point with an overall response rate of $28 \%$ (95\% CI: 20.7-37.0; $\mathrm{n}=36$ ); complete response (CR) rate was $12 \%(\mathrm{n}=15)$ and partial response $(\mathrm{PR})$ rate was $17 \%(\mathrm{n}=21)$ [23]. Median duration of response was 9.3 months (95\% CI: 4.8-23.0) for all responders and 23.0 months (95\% CI: 10.4-23.0) for patients in CR at a median follow-up time of 11.1 months [23]. Median OS was 9.1 months (95\% CI: 6.6-15.1) at a median follow-up time of 14.7 months and was not reached for patients in CR. The most common grade 3-4 adverse events (AEs) were thrombocytopenia (46\%), neutropenia (25\%), anemia (22\%), fatigue (11\%), hyponatremia $(8 \%)$ and nausea $(6 \%)$. Serious AEs (SAEs) occurred in $48 \%$ of patients, with the most common being pyrexia (7\%), pneumonia $(5 \%)$, sepsis $(5 \%)$ and fatigue $(4 \%)$. Selinexor was well tolerated, with treatment-emergent AEs managed with supportive care and/or dose modifications.

In addition to clinical efficacy and safety end points, patient-reported health-related quality of life (HRQoL) was an exploratory end point in SADAL and was assessed using the Functional Assessment of Cancer Therapy Lymphoma (FACT-Lym) questionnaire and the EuroQoL five-dimensions five-levels (EQ-5D-5L) instrument [23]. Previous reports have identified that DLBCL has a high humanistic burden and patients experience poor HRQoL resulting from disease symptoms and AEs related to treatment that interfere with physical, social and role functioning [24-27]. Furthermore, HRQoL declines with disease progression, and across increasing lines of therapy [27,28]. Patient-reported outcomes evidence such as HRQoL, along with clinical outcomes and survival benefit, are an important consideration in treatment decision making and contribute to a better understanding of the potential value of new therapies [29-35]. Disease-specific assessments such as the FACT-Lym can capture clinically-relevant, disease-specific symptomatic burden and disease-specific well-being [36-38]. In contrast, generic instruments such as the EQ-5D-5L can enable estimation of health utility scores, HRQoL relative to full health (i.e., score of 1) or 
death (i.e., score of 0) [39-41]. Health utility scores can be compared across diseases and applied in health economic analyses to evaluate outcomes such as quality-adjusted life years [39-41].

The objective of this study was to examine HRQoL and health utilities outcomes with single-agent selinexor in patients with RR DLBCL using patient-reported FACT-Lym and EQ-5D-5L data captured in the SADAL trial. The analyses used mixed effects models to evaluate longitudinal trends in HRQoL following initiation of selinexor, including minimal clinically meaningful differences (MCID), and examined the association between HRQoL and response to treatment. In addition, mixed-effects models were used to estimate health utility based on treatment response and derive health state utility values and disutilities associated with AEs/SAEs.

\section{Patients \& methods}

\section{Study design}

SADAL was a global, single-arm, open-label, Phase IIb study of single-agent selinexor in patients with RR DLBCL [42]. Full study details and patient eligibility criteria have been described elsewhere [23]. Briefly, eligible patients were $\geq 18$ years of age, with an estimated life expectancy of $>3$ months at study entry and had received two to five prior systemic regimens including at least one course of anthracycline-based chemotherapy and at least one course of anti-CD20 immunotherapy. Patients had pathologically confirmed de novo DLBCL or DLBCL transformed from previously diagnosed indolent lymphoma. Patients were excluded if they had primary mediastinal B-cell lymphoma, DLBCL with meningeal involvement, or known CNS lymphoma, or were eligible for high-dose therapy with ASCT. The study protocol was approved by the institutional review board or an independent ethics committee at each study center. Oral selinexor $(60 \mathrm{mg})$ was administered as a single agent on days 1 and 3 of each week (bi-weekly [BIW]) until disease progression, death or unacceptable toxicities.

\section{Data collection, patient-reported HRQoL \& health utility assessments}

Patients self-administered and completed the FACT-Lym (version 4) questionnaire and the EQ-5D-5L instrument. Data were collected at baseline (screening visit 2), on day 1 of each 28-day treatment cycle beginning at cycle 2, and at end of treatment (EOT; i.e., time of drug discontinuation). FACT-Lym is an instrument that has been previously applied to measure HRQoL in patients with B-cell malignancies [43-46], including RR DLBCL [47]. FACT-Lym combines the general version of the FACT, in other words, FACT - General (FACT-G; 27 items in four primary domains) with a lymphoma subscale (Lym domain; 15 items) [36,37]. The Lym domain addresses lymphoma-specific well-being in terms of symptomatic burden and impact of treatments. The FACT-Lym total score (TS; score range: $0-168)$ is obtained by adding individual subscale scores for physical well-being (7 items), social/family well-being (7 items), emotional well-being ( 6 items) and functional well-being domains ( 7 items) of the FACT-G (score range: 0-108) and the Lym domain (score range: 0-60). The FACT-Lym Trial Outcome Index (TOI) is comprised of the physical and functional subscales of the FACT-G and the Lym domain (score range: 0-116). Higher scores for the FACT-G, FACT-Lym TOI, and FACT-Lym TS indicate better HRQoL [36,37]. Assessment completeness was defined according to the FACT-Lym Scoring Guidelines (version 4), which allows subscales to be calculated if $>50 \%$ of items are present, and TSs if $>80 \%$ of items are present $[36,37$.

Health utility in SADAL was captured with the EQ-5D-5L instrument. The EQ-5D-5L instrument includes dimensions of mobility, self-care, usual activities, pain/discomfort and anxiety/depression, each with five response levels $[41,48]$. Response for each dimension is coded as a single-digit number where level 1 equates to no problems and level 5 to extreme problems. Combined, the five dimensions create a five-digit score that corresponds to the health state. Data were scored using a crosswalk between patient profiles (i.e., five-digit score) and the EQ-5D-5L index was calculated based on the US standard value set [40]. As a result, data for each of the five dimensions were required to be assessed as complete for inclusion in the analysis and to enable derivation of a final index value. It should be noted that patient-reported visual analog scale data were not captured in the SADAL trial.

\section{Statistical analysis}

Number of patients \& patient characteristics

The HRQoL dataset included patients who received at least one $60-\mathrm{mg}$ dose of selinexor in the mITT population $(\mathrm{n}=127)$, had complete data from baseline and at least one follow-up visit, and had complete baseline prognostic variable data. The analysis considered covariates of sex, age, race, ethnicity and Eastern Cooperative Oncology Group performance status (ECOG PS) for inclusion in the longitudinal models. Patients with missing ECOG PS at baseline, which was found to be a statistically significant covariate, were omitted from the HRQoL and health utility analyses. Treatment responders corresponded to patients with objective response (CR or PR) in SADAL 
according to the 2014 Lugano criteria for response assessment of lymphoma by independent central review [42,49]. In analyses stratified by best overall treatment response status, patients who did not have evaluable response were excluded. For the FACT-Lym dataset, patient visits with $<80 \%$ of items answered for the TS and $<50 \%$ for subscales were excluded from the analysis. Cycles with $<20 \%$ of the sample were not included in the analysis (e.g., unscheduled visits) to limit bias which could result from small sample sizes. For the health utility dataset, cycles after eight were excluded due to having $<20 \%$ of patients, with data at baseline, remaining on treatment.

\section{Longitudinal regression analysis}

Longitudinal models were constructed to evaluate the magnitude of change from baseline for the FACT-G, FACTLym TOI, FACT-Lym TS and EQ-5D-5L health utility for each follow-up cycle. Mixed-effects linear regression models were constructed allowing for random slope and intercept terms. Categorical time (i.e., cycle) was treated as a fixed and random effect. Univariate, multivariate and stepwise techniques were tested to identify covariates that improved fit. Models were adjusted for baseline HRQoL score and relevant baseline patient characteristics that improved fit $(\mathrm{p}<0.10)$; nonsignificant $(\mathrm{p}>0.10)$ covariates were excluded. Only final adjusted models which include baseline scores and specified baseline characteristics that improved fit are reported.

\section{Minimal clinically important differences}

Estimated differences in scores based on treatment response status were compared against MCID thresholds obtained from the literature. An MCID represents the minimum clinically meaningful change in patient-reported HRQoL scores for a validated instrument, and may be interpreted as the minimum level of perceived benefit with a therapy option by the patient [50,51]. MCIDs were identified through a targeted literature search for previously validated ranges for the lower and upper thresholds determined through distribution- and anchor-based methods. MCID ranges were 3-7 for the FACT-G, 5.5-11 for the FACT-Lym TOI and 6.5-11.2 for the FACT-Lym TS [47]. For the EQ-5D-5L, Pickard et al. [52] recommended a threshold of 0.06 for identifying MCID in US-index scores, while Kvam et al. [53] suggested the threshold range of 0.08-0.1. Based on these two previous validation studies, the analysis herein considered 0.06 as the lower threshold limit of MCID and 0.1 as the upper limit. The proportions of patients experiencing HRQoL or health utility improvement, maintenance or decline based on the high and low MCID limits were calculated for each score at each cycle. Patients having an increase in score that exceeded the MCID threshold were considered as having clinically meaningful improvement, while those having a decrease greater than the MCID threshold were considered as having clinically meaningful decline. Patients with change less than the MCID were considered stable.

\section{Responder analysis}

Differential attrition rates were observed between responders and nonresponders which motivated the use of mixed-effects models rather than pairwise t-tests by cycle visit. The mixed-effect models assumed data are missingat-random, which is less robust than an assumption of missing completely at random as in pairwise analysis. The missing-at-random assumption was tested by examining patterns of missingness in the trial data. Unlike the longitudinal regression models which treated time as categorical, the responder analysis considered time as a continuous variable to account for varying EOT visit times. This approach was required to ensure that comparisons between responders and nonresponders were not confounded by differential discontinuation dates. The mixedeffects models included a random intercept, time as a fixed and random effect, baseline score and adjusted models included ECOG PS as a covariate.

The interaction effect of treatment response (i.e., objective response or no response) and time was estimated to compare the differences in FACT-G, FACT-Lym TOI, FACT-Lym TS and EQ-5D-5L index scores based on responder status over the course of follow-up. An interaction effect of treatment and time was considered present based on a significance level of 0.05 for the interaction coefficient. Mean differences in scores were estimated directly from the mixed effects model at each cycle. In the analysis, a positive estimated mean difference implies that treatment responders had higher relative HRQoL than nonresponders at that cycle. An interaction term between responder status and time was introduced in all responder analysis where the interaction was statistically significant to minimize the risk of bias associated with differential attrition. Estimated differences in scores based on treatment response status were compared against MCID thresholds obtained from the literature. 


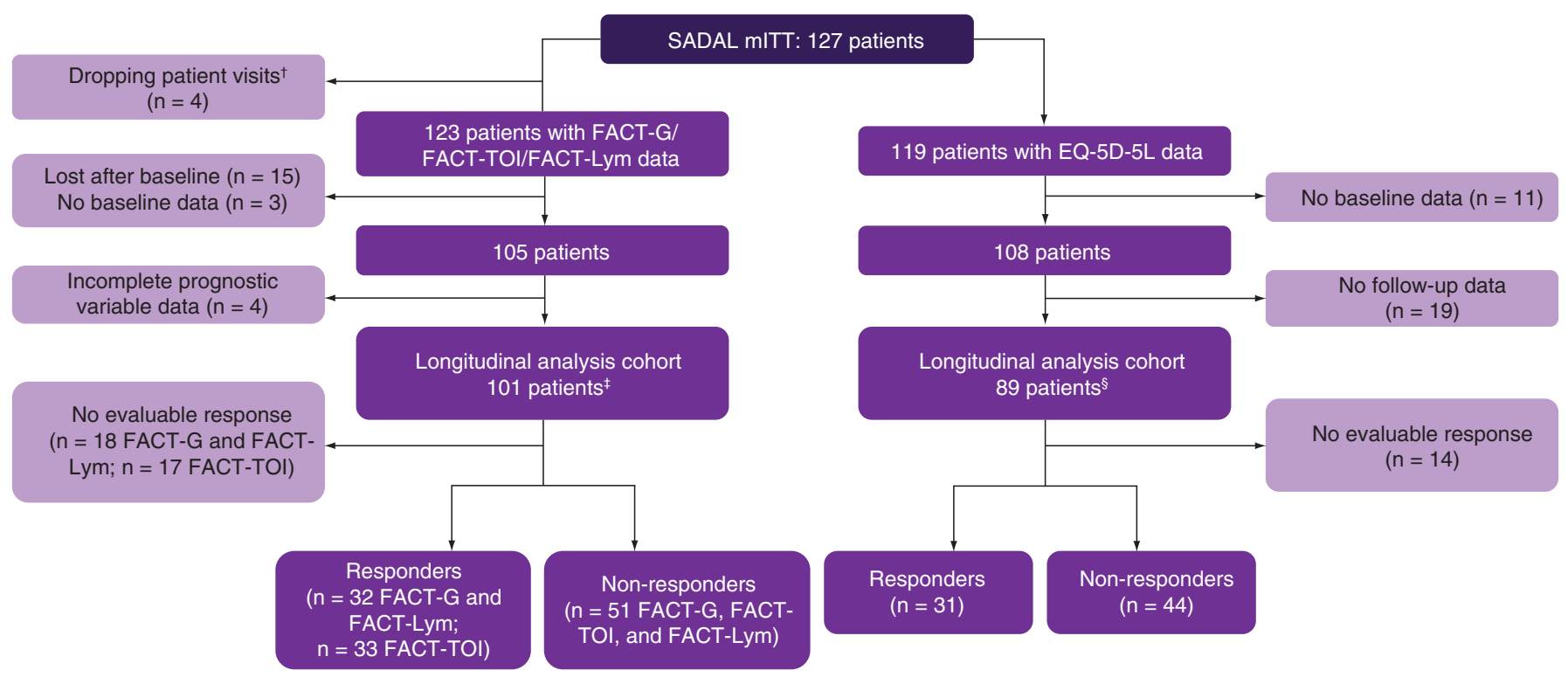

Figure 1. Study flowchart (patient disposition).

† Patient visits were dropped if $<80 \%$ of items were answered for the total score, if $<50 \%$ of items were answered for the subscale, if they were unscheduled visits, or if $<20 \%$ of the sample answered the questionnaire at that cycle (the whole cycle was dropped in this case). $¥$ The longitudinal analysis cohort required baseline health-related quality of life data, at minimum one follow-up health-related quality of life data data and Eastern Cooperative Oncology Group performance status data at baseline.

$\S$ The longitudinal analysis cohort required baseline data and at minimum one follow-up EQ-5D-5L assessment.

EQ-5D-5L: EuroQoL five-dimensions five-levels; FACT-G: Functional Assessment of Cancer Therapy - general; FACT-TOI: FACT - Lymphoma Trial Outcome Index; FACT-Lym: FACT - lymphoma.

\section{Health state utility values of disutility of AEs}

The analysis sought to evaluate how utility values differed for patients based on response to selinexor treatment and disease progression. Three health states of progressive disease, stable disease and response were considered, which were derived based on best overall response as assessed by an independent central review panel. Each patient was considered to have stable disease at baseline. At cycle 2, patients were categorized based on the best overall response. Mixed effects linear regression models were constructed with the EQ-5D-5L index as the outcome. A random intercept was included and categorical time (i.e., cycle) was treated as a fixed and random effect. Mean values for each disease state at baseline and EOT were estimated. The coefficients for progression representing the mean differences between health states were estimated with 95\% CIs and p-values.

The disutilities of aggregate and individual grade $\geq 3$ AEs and SAEs with $\geq 10 \%$ prevalence in SADAL were analyzed. A disutility represents a loss in EQ-5D-5L utility score (i.e., decrease in HRQoL) that is associated with experiencing an AE/SAE. All models included the baseline EQ-5D-5L index score and coefficients, 95\% CIs, and p-values were reported as adjusted for health states (i.e., disease progression). Due to the small sample sizes and patient attrition, the change associated with AEs was estimated based on all postbaseline cycles.

\section{Results}

\section{Patient characteristics}

From the mITT population $(\mathrm{n}=127)$, the QoL dataset included 105 patients for the FACT-G and FACT-Lym TOI, and 104 patients for the FACT-Lym TS who had completed enough items at baseline and at least one follow-up treatment cycle (Figure 1). Following exclusion of patients with no baseline ECOG PS data, 101 patients were retained in the longitudinal analysis cohort for each score. A total of 83 patients with complete FACT-G and FACT-Lym TS data had an evaluable response, of whom 32 were treatment responders and 51 were nonresponders. Similarly, 84 patients with complete FACT-Lym TOI had an evaluable response, with 33 treatment responders and 51 nonresponders. In the HRQoL dataset $(\mathrm{n}=101)$, mean age was 66.3 (standard deviation [SD]: 10.4; range: $35-87)$ and $58 \%$ of patients were male $(\mathrm{n}=59)$ (Table 1). The proportions of patients with ECOG PS of 0,1 and 2 were $48 \%(n=48), 45 \%(n=46)$ and $8 \%(n=8)$, respectively. 
Table 1. Baseline characteristics of patients in the health-related quality of life and health utility datasets.

\begin{tabular}{|c|c|c|}
\hline Characteristic & HRQoL dataset value & Health utility dataset value \\
\hline $\mathrm{N}$ & 101 & 89 \\
\hline Male & $59(58 \%)$ & $48(53.9 \%)$ \\
\hline Mean age (SD), years & $66.3(10.4)$ & $66.1(10.3)$ \\
\hline \multicolumn{3}{|l|}{ Age group $(n, \%)$ : } \\
\hline$-18-50$ & $7(7 \%)$ & $6(6.7 \%)$ \\
\hline$-51-64$ & $33(33 \%)$ & $29(32.6 \%)$ \\
\hline$-\geq 75$ & $25(24 \%)$ & $20(22.5 \%)$ \\
\hline \multicolumn{3}{|l|}{ Race $(n, \%)$ : } \\
\hline -White & $79(78 \%)$ & $69(77.5 \%)$ \\
\hline - Asian & $10(10 \%)$ & $10(11.2 \%)$ \\
\hline - Other & $6(6 \%)$ & $4(4.5 \%)$ \\
\hline - Unknown & $4(4 \%)$ & $3(3.4 \%)$ \\
\hline - Not reported & $11(11 \%)$ & $10(11.2 \%)$ \\
\hline - Missing & $2(2 \%)$ & - \\
\hline \multicolumn{3}{|l|}{ ECOG PS: } \\
\hline-0 & $48(48 \%)$ & $42(47.2 \%)$ \\
\hline-1 & $45(46 \%)$ & $41(46.1 \%)$ \\
\hline-2 & $8(8 \%)$ & $5(5.6 \%)$ \\
\hline - Missing & 0 & $1(1.1 \%)$ \\
\hline
\end{tabular}

Similarly, from the mITT population of SADAL $(n=127)$, the health utility dataset included 89 patients with EQ-5D-5L index score at baseline and at least one follow-up cycle (Figure 1). Among patients in the health utility dataset, the mean age was 66.1 years $(S D=10.3)$ and $53.9 \%$ of patients were male $(n=48)$ (Table 1$)$. The proportions of patients with ECOG PS of 0, 1 and 2 were $47.2 \%(n=42), 46.1 \%(n=41)$ and 5.6\% $(n=5)$, respectively. A total of 75 patients with complete EQ-5D-5L data had an evaluable response, of whom 31 had objective response and 44 were nonresponders.

It should be noted that baseline characteristics of included patients and excluded patients were largely similar in the two cohorts. One noteworthy exception was that the proportion of excluded patients with ECOG PS of 2 was higher versus included patients in both the HRQoL (25 vs 8\%) and the EQ-5D-5L (27.3 vs 5.6\%) cohort. Furthermore, more men were excluded from the EQ-5D-5L cohort (68.2 vs 53.9\%).

HRQoL analyses

Results from the mixed-effects regression analysis for the FACT-G, FACT-Lym TOI and the FACT-Lym TS are shown in Figure 2A-C. Model coefficients are presented in Supplementary Table 1. Final optimized models were adjusted for baseline scores and baseline ECOG PS $(0,1$ or 2). The number of patients remaining in the HRQoL dataset declined with each cycle. HRQoL decreased across all three scores between baseline and the final visit with differences of -6.0 (95\% CI: -9.2 to -2.8$)$, -8.6 (95\% CI: -13.0 to -4.3$)$ and -9.9 (95\% CI: -15.5 to -4.3 ) for FACT-G, FACT-Lym TOI and FACT-Lym TS, respectively. Observed decreases from baseline were significant at early cycles of treatment and at the EOT.

Health utility analyses

Results of the mixed-effects regression analysis are presented in Figure 2D, with model coefficients indicated in Supplementary Table 2. Patients had a mean of four assessments (range: 2-9). The number of patients remaining in the health utility dataset declined from baseline to cycle 8. Final optimized models were adjusted for baseline EQ5D-5L index scores and baseline ECOG PS $(0,1$ or 2$)$, which was identified as a significant covariate $(\mathrm{p}<0.01)$. 
(A)

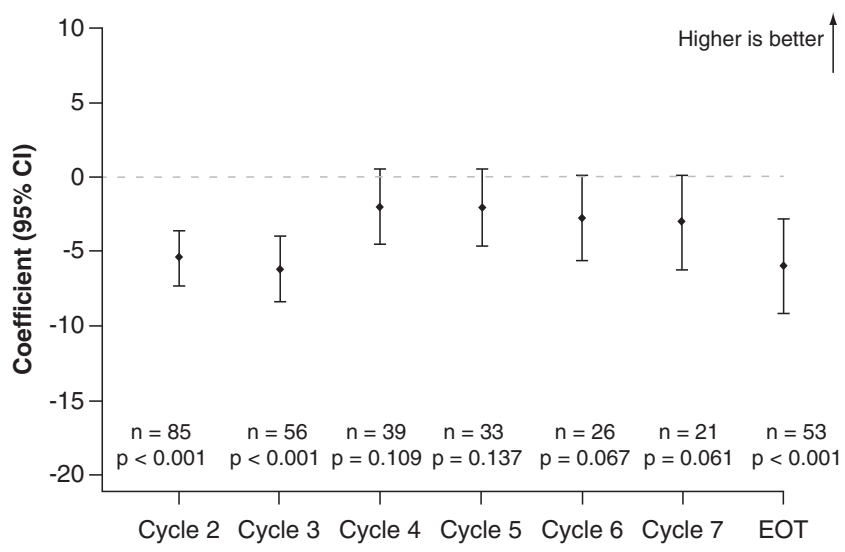

(C)

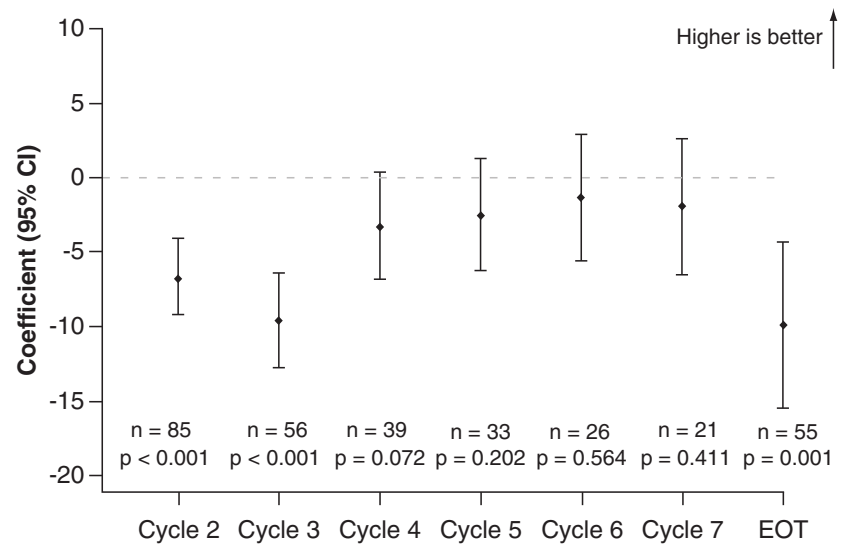

(B)

FACT-TOI

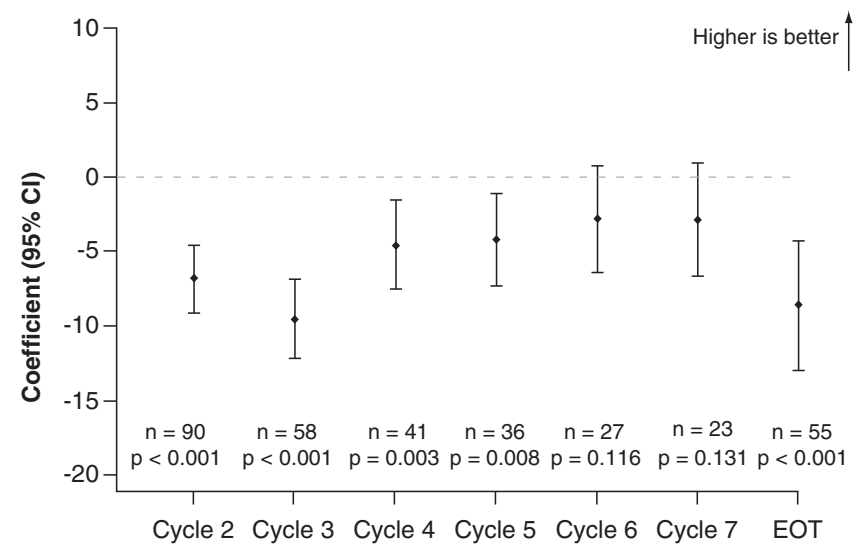

(D)

EQ-5D-5L

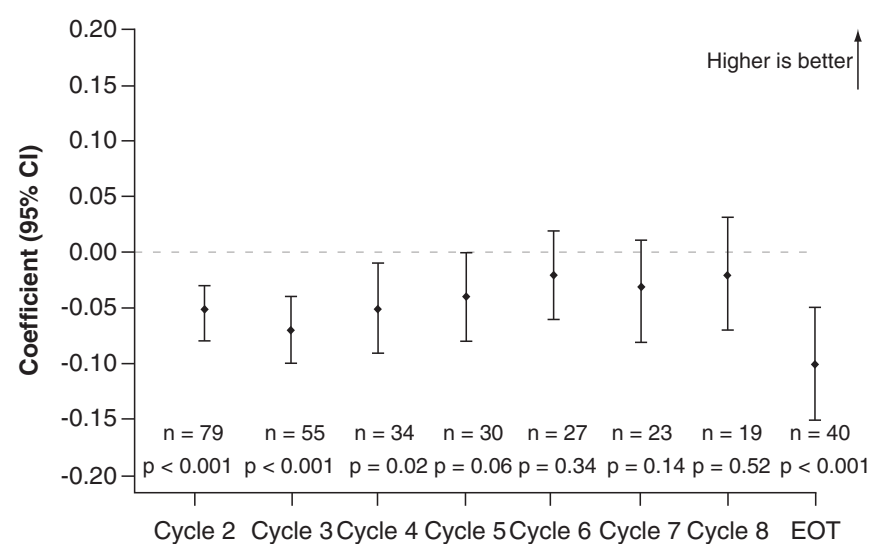

Figure 2. Longitudinal changes in quality of life and health utility. Longitudinal change in health-related quality of life as evaluated by mixed-effects regression models for (A) Functional Assessment of Cancer Therapy - General, (B) Functional Assessment of Cancer Therapy - Lymphoma Trial Outcome Index, (C) Functional Assessment of Cancer Therapy - Lymphoma total score and (D) EuroQoL five-dimensions five-levels index score. Models were adjusted for baseline score and Eastern Cooperative Oncology Group performance status. EOT: End of treatment; EQ-5D-5L: EuroQoL five-dimensions five-levels; FACT-G: Functional Assessment of Cancer Therapy - general; FACT-TOI: FACT - Lymphoma Trial Outcome Index; FACT-Lym: FACT - lymphoma.

EQ-5D-5L index scores decreased from baseline at each cycle. Observed decreases were significant in cycles 2-4, and at the EOT $(-0.10$ [95\% CI: -0.15 to -0.05$]$; $\mathrm{p}<0.001)$. Majority of patients experienced no clinically meaningful change in the EQ-5D-5L index score from baseline through to cycle 8 based on the lower and upper MCID thresholds of 0.06 and 0.1 , respectively.

\section{Minimal clinically important differences}

The proportions of patients achieving clinically important change in HRQoL according to each score were evaluated based on MCID thresholds reported in literature. Based on the MCID lower limit, majority of patients experienced decline in FACT-G (Figure 3A; Supplementary Table 3), FACT-Lym TOI (Figure 3B; Supplementary Table 4) and FACT-Lym TS (Figure 3C; Supplementary Table 5) during the early cycles of treatment and EOT. Based on the MCID upper limit, majority of patients experienced no change or improvement in FACT-G, FACT-Lym TOI and FACT-Lym TS compared with baseline for early cycles but experienced decline at the EOT. For the EQ-5D-5L index score, the majority of patients experienced no change or improvement from baseline according to the lower and upper MCID limit (Figure 3D). 
(A)

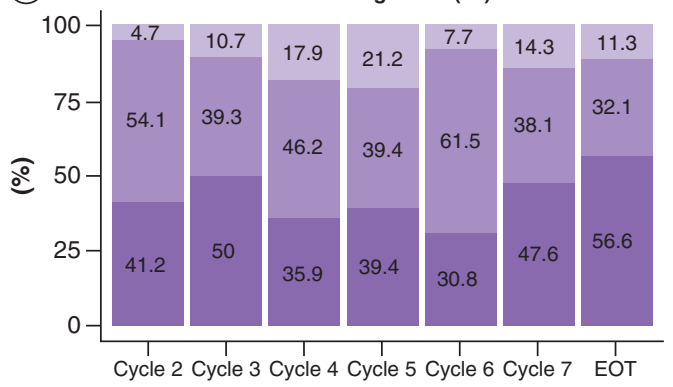

(B)

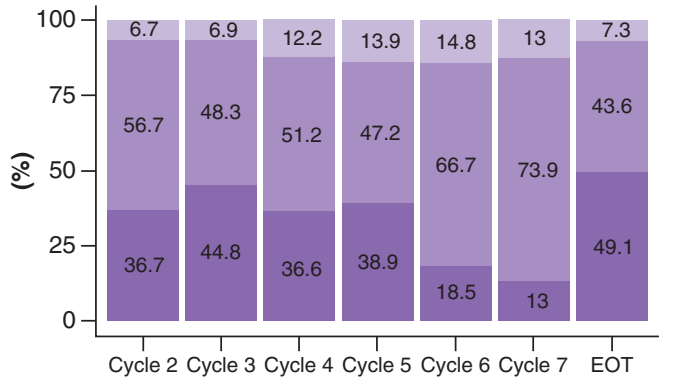

(C)

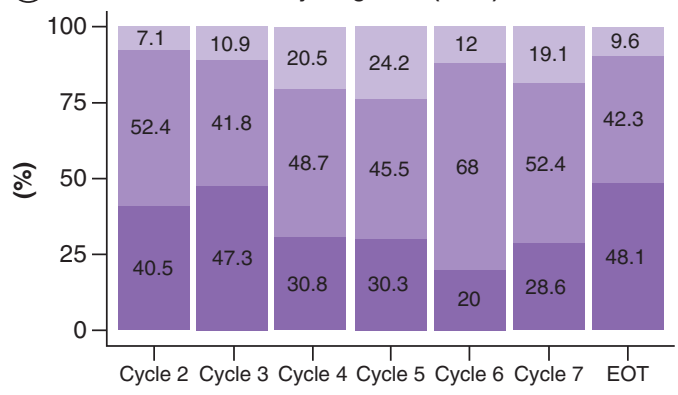

(D)

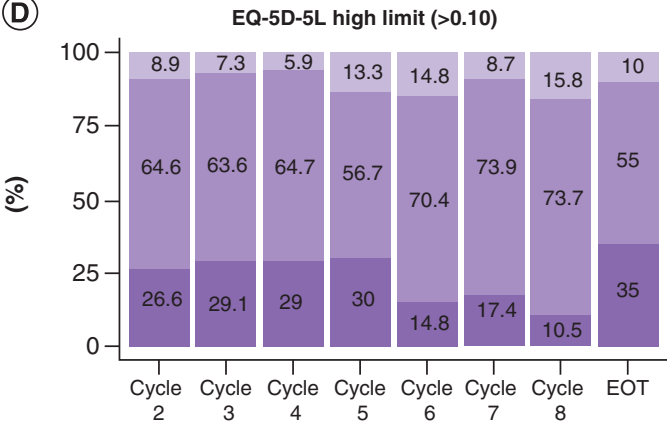

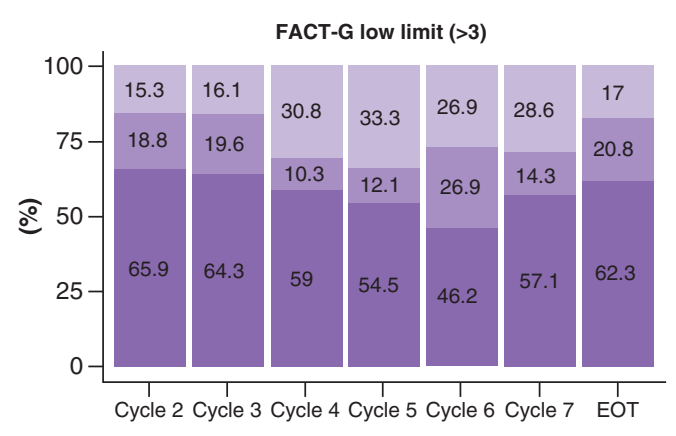

FACT-TOI low limit (>5.5)

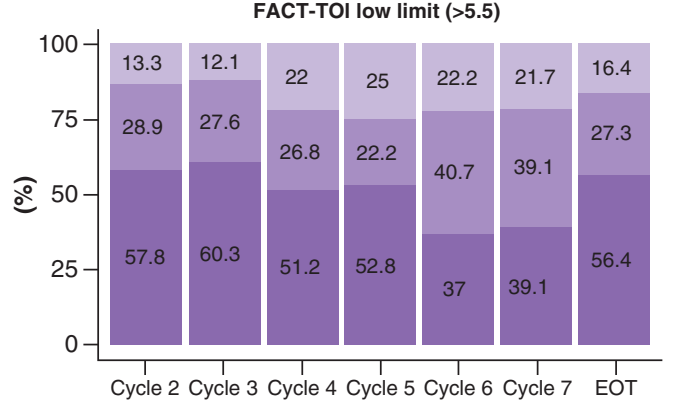

FACT-Lym low limit (>6.5)

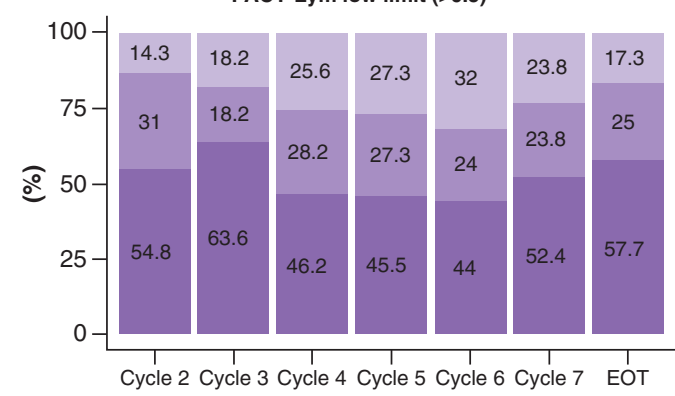

EQ-5D-5L low limit (>0.06)

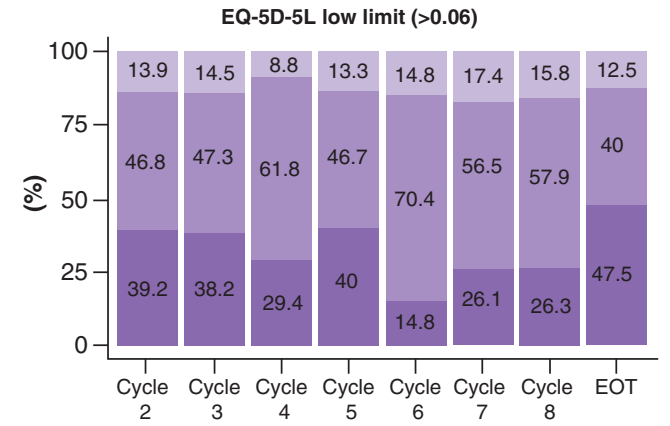

Improvement

No change

Decline

Figure 3. Proportion of patients with decline, no change and improvement in health-related quality of life scores based on the lower and upper limit of minimal clinically important difference thresholds for (A) Functional Assessment of Cancer Therapy - General, (B) Functional Assessment of Cancer Therapy - Lymphoma Trial Outcome Index, (C) Functional Assessment of Cancer Therapy - Lymphoma total score and (D) EuroQoL five-dimensions five-levels index score.

EOT: End of treatment; EQ-5D-5L: EuroQoL five-dimensions five-levels; FACT-G: Functional Assessment of Cancer Therapy - general; FACT-TOI: FACT - Lymphoma Trial Outcome Index; FACT-Lym: FACT - lymphoma. 
(A)

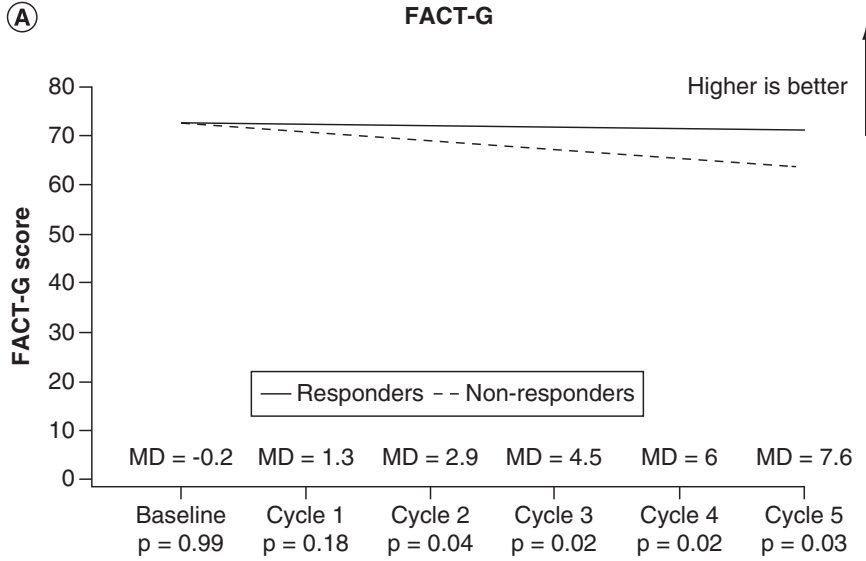

(B)

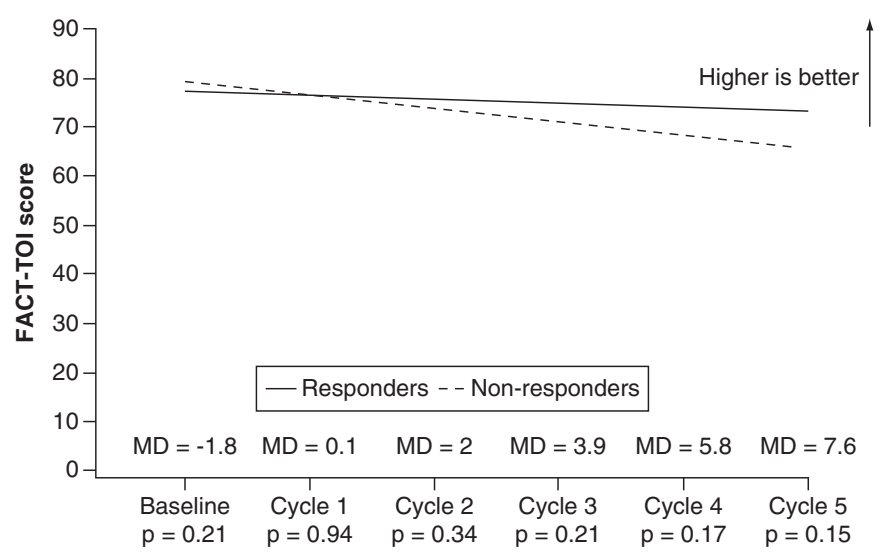

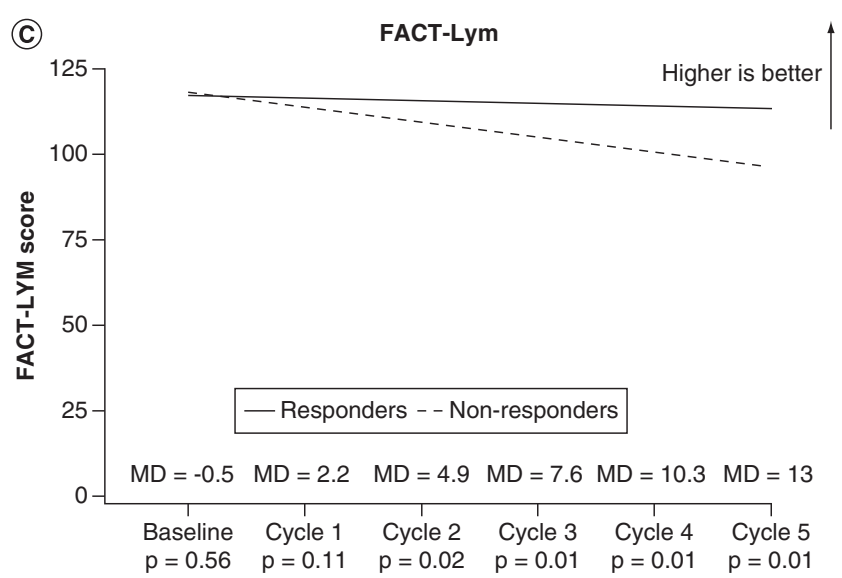

(D)

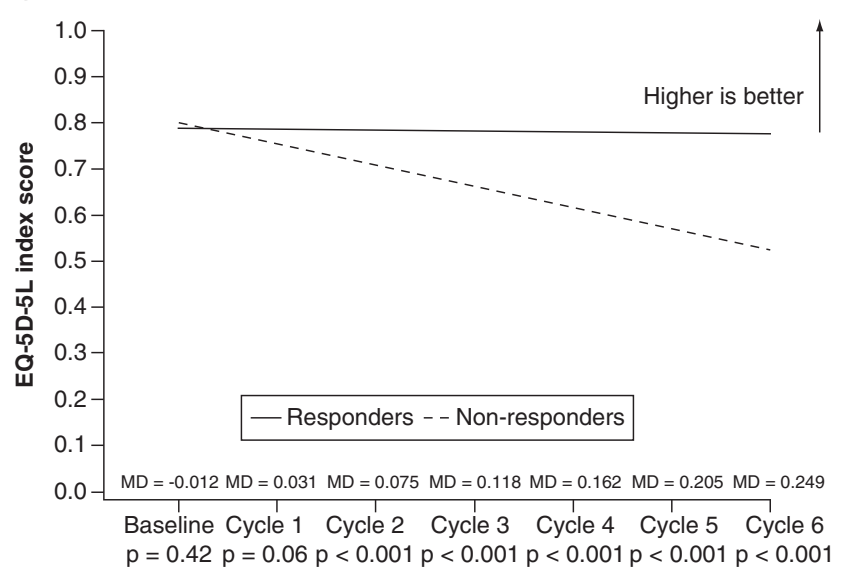

Figure 4. Longitudinal health-related quality of life difference analysis - mean differences in health-related quality of life scores between objective responders and nonresponders for (A) Functional Assessment of Cancer Therapy - General, (B) Functional Assessment of Cancer Therapy - Lymphoma Trial Outcome Index, (C) Functional Assessment of Cancer Therapy - Lymphoma total score and (D) EuroQoL five-dimensions five-levels index score. Models were adjusted based on baseline Eastern Cooperative Oncology Group performance status. A positive estimated mean difference implies that responders had higher relative health-related quality of life than nonresponders at that cycle.

EQ-5D-5L: EuroQoL five-dimensions five-levels; FACT-G: Functional Assessment of Cancer Therapy - general; FACT-TOI: FACT - Lymphoma Trial Outcome Index; FACT-Lym: FACT - lymphoma; MD: Mean difference.

\section{Responder analysis}

Model-based estimated mean differences in HRQoL scores between treatment responders and nonresponders are presented in Figure 4A-C for the three scores at baseline and follow-up cycles. Predicted HRQoL means of the responder analysis are presented in Supplementary Table 6 for the FACT-G, Supplementary Table 7 for the FACTLym TOI and Supplementary Table 8 for the FACT-Lym TS. Results of the responder analysis showed a significant interaction between responder status and time for the FACT-G $(p=0.05)$ and FACT-Lym TS $(p=0.05)$, while the interaction was not significant for FACT-Lym TOI $(\mathrm{p}=0.10)$. While the interaction was not significant for the FACT-Lym TOI, the difference in mean scores was similar to the FACT-G over six cycles; therefore, the lack of observed significance could be caused by higher variability in patient responses. At baseline, scores for the FACT G, FACT-Lym TOI and FACT-Lym TS were similar for responders and nonresponders, indicating no significant differences in HRQoL. However, responders had higher scores by cycle 2 for the FACT-G, FACT-Lym TOI and FACT-Lym TS. The differences in scores between responders and nonresponders became significant $(\mathrm{p} \leq 0.05)$ by cycle 3 for the FACT-G and the FACT-Lym TS (Figure 4), denoting higher HRQoL among responders. Observed differences increased with each cycle, indicating that treatment responders maintained a higher HRQoL over a longer period of time while nonresponders declined rapidly. Nonresponders experienced decline in scores from 
Table 2. Estimated EuroQoL five-dimensions five-levels health state utility values.

\begin{tabular}{|lll|}
\hline Health status & Baseline estimated mean $(95 \% \mathrm{Cl})$ & End of treatment estimated mean $(95 \% \mathrm{Cl})$ \\
\hline Progressive disease & $0.731(0.668-0.793)$ & $0.669(0.619-0.719)$ \\
\hline Stable disease & $0.783(0.756-0.809)$ & $0.721(0.658-0.784)$ \\
\hline Response & $0.801(0.741-0.861)$ & $0.739(0.689-0.790)$ \\
\hline Pairwise comparisons & Difference $(95 \% \mathrm{Cl})$ & $\mathrm{p}$-value \\
\hline Progressive vs stable disease & $-0.052(0.004$ to -0.109$)$ & 0.073 \\
\hline Response vs progressive disease & $0.07(0.029-0.111)$ & 0.001 \\
\hline Response vs stable disease & $0.018(-0.035-0.072)$ & 0.507 \\
\hline
\end{tabular}

baseline that exceeded the lower MCID threshold by cycle 3 for the FACT-G ( $\geq 3$, Supplementary Table 6 ), and by cycle 4 for the FACT-TOI ( $\geq 5.5$, Supplementary Table 7) and the FACT-Lym TS ( $\geq 6.5$, Supplementary Table 8). Furthermore, the observed declines exceeded the upper MCID thresholds by cycle 5 for the FACT-G $(\geq 7)$ and FACT-Lym TS $(\geq 12.5)$ and cycle 6 for the FACT-TOI ( $\geq 5.5)$. In responders, changes in scores from baseline did not exceed the lower or upper limit of the MCIDs for any score at any follow-up cycle.

Likewise, model-based estimated mean differences in EQ-5D-5L index scores between treatment responders $(\mathrm{n}=31)$ and nonresponders $(\mathrm{n}=44)$ at baseline and follow-up cycles are presented in Figure 4D. Predicted EQ-5D-5L means of the responder analysis are presented in Supplementary Table 9. The mixed-effects models, adjusted for baseline ECOG PS, revealed a significant interaction between time and responder status ( $\mathrm{p}=0.002)$. Baseline EQ-5D-5L index scores were similar for responders and nonresponders suggesting a similar health utility. By cycle 2, treatment responders had higher estimated mean scores. This difference became significant by cycle 3 (mean difference: 0.075 [95\% CI: 0.023-0.126]; $\mathrm{p}<0.001$ ), and was maintained through to cycle 7 (mean difference: 0.249 [95\% CI: 0.095-0.402]; $\mathrm{p}<0.001$ ). Treatment responders did not experience a decline in scores that exceeded the lower or upper threshold of MCIDs at any cycle. Nonresponders experienced decline in scores from baseline, where the lower threshold limit of MCID was exceeded by cycle 3, and upper threshold limit of MCID was exceeded by cycle 4 .

\section{Health state utility}

Estimated mean health state utility values at the EOT were 0.669 (95\% CI: 0.619-0.719) for progressive disease, 0.721 (95\% CI: 0.658-0.784) for stable disease and 0.739 (95\% CI: 0.689-0.790) for treatment response (Table 2). Pairwise analysis identified that patients with stable disease had numerically superior health state utility compared with patients with progressive disease, however the difference was not significant $(p=0.073)$. Responders had significantly higher health state utility compared with patients with progressive disease $(p=0.001)$ but not stable disease $(p=0.507)$. The estimated mean difference between health states of treatment response and progressive disease exceeded the lower threshold of the MCID of 0.06 (mean difference: 0.07 [95\% CI: 0.029-0.111]).

\section{Disutility analysis}

Mixed-effects regression models were adjusted for disease progression to estimate disutilities associated with AEs and SAEs with $>10 \%$ incidence in SADAL (Table 3). In the EQ-5D-5L analysis cohort, $88 \%$ of patients experienced at least one grade $\geq 3 \mathrm{AE}$, with $47 \%$ of patients experiencing at least one SAE. Having at least one grade $\geq 3 \mathrm{AE}$ resulted in a significant reduction in the EQ-5D-5L index, with a mean difference of -0.036 (95\% CI: -0.067 to -0.006; $\mathrm{p}=0.02$ ). Individual AEs were generally negatively associated with the EQ-5D-5L index score, and significant negative associations were observed for any grade $\geq 3 \mathrm{AE}$ and metabolism and nutrition disorders. Neutropenia and anemia were associated with a nonsignificant, minor positive association. Having at least one SAE was also associated with a significant decrease in the EQ-5D-5L index score with a mean difference of -0.038 ( $95 \%$ CI: -0.062 to $-0.013 ; \mathrm{p}=0.002)$. The only SAE with $>10 \%$ incidence was infections and infestations $(22 \%)$. While the association for infections and infestations and EQ-5D-5L was negative, the effect was not significant.

\section{Discussion}

HRQoL evidence is increasingly important in healthcare decision making and complements clinical and safety outcomes in evaluating the benefit-risk balance of new oncology treatments [29-35]. Patients with DLBCL experience a substantial burden of disease arising from both disease symptoms and treatment-related AEs, and declining 


\begin{tabular}{|c|c|c|c|c|}
\hline EQ-5D-5L cohort $(n=89)$ & Incidence & Coefficient & $95 \% \mathrm{Cl}$ & p-value \\
\hline At least one grade $\geq 3 \mathrm{AE}$ & $78(88 \%)$ & -0.038 & -0.062 to -0.013 & 0.002 \\
\hline Blood and lymphatic system disorders: & $78(88 \%)$ & -0.025 & $-0.051-0.001$ & 0.07 \\
\hline - Thrombocytopenia & $57(64 \%)$ & -0.009 & $-0.038-0.020$ & 0.54 \\
\hline - Anemia & $27(30 \%)$ & 0.000 & $-0.039-0.039$ & 0.99 \\
\hline Gastrointestinal disorders & $23(26 \%)$ & -0.042 & $-0.090-0.006$ & 0.09 \\
\hline Fatigue & $13(15 \%)$ & -0.055 & $-0.119-0.010$ & 0.10 \\
\hline Metabolism and nutrition disorders & $9(10 \%)$ & -0.082 & -0.120 to -0.044 & $<0.001$ \\
\hline Infections and infestations & $20(22 \%)$ & -0.017 & $-0.059-0.024$ & 0.42 \\
\hline
\end{tabular}

HRQoL with disease progression and across increasing lines of therapy [24-27]. In one United Kingdom real-world study, DLBCL-specific health state utility values were estimated to decrease from 0.81 (standard error $=0.016$ ) in first-line of therapy to 0.59 (standard error $=0.093$ ) in third and further line of therapy as assessed with the EQ-5D-5L [27]. Well-tolerated oral therapies could limit the potential humanistic burden associated with multidrug injectable regimens among transplant ineligible patients with RR DLBCL.

The current analysis examined HRQoL and health utility impacts associated with oral, single-agent selinexor in the SADAL study population of heavily pretreated patients with RR DLBCL who were not candidates for transplantation (ASCT or CAR T-cell therapy) or experienced relapse after ASCT [23]. In the mITT population, longitudinal mixed-effects models identified decline in HRQoL from baseline, which were significant in the early cycles and at EOT. The clinically meaningful decline from baseline was greater than the MCID lower limit for the FACT-G, FACT-Lym TOI and the FACT-Lym TS at all time points. Similarly, EQ-5D-5L index scores decreased significantly from baseline to EOT, which is reflective of the heavily pretreated and advanced nature of RR DLBCL among patients included in the analysis population.

The analysis also sought to identify HRQoL trends among treatment responders and nonresponders, given the study population has poor prognosis and treatment should seek to delay HRQoL changes for as long as possible. While HRQoL declined in the mITT population overall, patients who had CR or PR maintained higher HRQoL over a longer period of time compared with patients who did not respond to treatment. Objective responders did not experience clinically meaningful change in HRQoL from baseline during the follow-up cycles based on FACT-G, FACT-Lym TOI or FACT-Lym TS scores according to the lower and upper limit of MCIDs. Similarly, treatment responders had significantly higher EQ-5D-5L index scores than nonresponders by cycle 3 and experienced no clinically meaningful change in health utility from baseline at any cycle. In contrast, nonresponders experienced rapid, clinically meaningful HRQoL and health utility decline.

Stable disease and treatment response were associated with higher mean health utility compared with progressive disease. The estimated difference in utility between treatment response and progressive disease was both statistically significant and clinically meaningful as it exceeded the lower threshold of the MCID of 0.06 (mean difference: 0.07; $\mathrm{p}=0.001$ ). In addition, grade $\geq 3$ AEs and SAEs were associated with decrements in health utility, which did not reach the lower threshold of the MCID to be assessed as clinically meaningful. Collectively, these analyses suggest that single-agent selinexor is associated with HRQoL maintenance that is consistent with durable clinical response and delayed disease progression in patients with objective response. In treatment responders (PR or CR), the median OS was not reached while the median duration of response was 23 months (95\% CI: 10.4-23.0) for patients with CR and 4.4 months (95\% CI: 2.0 to not estimable) for patients with PR [23]. As median progression-free survival was 2.6 months (95\% CI: 1.9-4.0), for nonresponders who experienced disease progression, duration of HRQoL impact was short. 
Several interventional studies have examined patient-reported HRQoL and health utility in the third-line RR DLBCL setting, including several recent reports examining CAR-T therapies [12,47,54-63]. In a recent report, clinically meaningful improvements in HRQoL from baseline at 3, 6, 12 and 18 months were observed in patients achieving CR or PR with tisagenlecleucel in the JULIET trial according to FACT-G, FACT-Lym TS and FACT-Lym TOI [47]. EQ-5D-3L mapped from patient-reported Short-Form 36 data in the JULIET trial of tisagenlecleucel corresponded to health state utility values of 0.83 for progression-free disease and 0.71 for progressed disease [59,60]. Similarly, patients had clinically meaningful improvements at 6 and 12 months compared with baseline in European Organization for the Research and Treatment of Cancer Quality of Life QuestionnaireCore 30 global health, physical function, fatigue and pain scores with lisocabtagene maraleucel treatment in the TRANSCEND NHL 001 trial [54,55]. EQ-5D-5L mean preference-weighted scores declined from baseline to month 1 (mean difference: -0.016 [SD $=0.144]$ ), then increased through to month 6 (mean difference: 0.019 [SD $=0.133]$ ) [61]. At 6 months, 30\% of patients experienced improvement in health utility that exceeded an MCID threshold of $\geq 0.07$ [54]. In contrast to eventual improvement in HRQoL and health utility observed with CAR T-cell therapies, HRQoL and EQ-5D-5L index scores decreased from baseline to EOT for patients in SADAL. This trend may be reflective of baseline characteristics and prognostic factors such as age, ECOG PS and baseline HRQoL, within the transplant-ineligible patient population of SADAL that differ from study populations of CAR T-cell therapies.

The present study has several limitations. As SADAL was a single-arm study with no comparator arm, treatmentassociated changes in HRQoL or health utility cannot be directly tested from the clinical trial data. In addition, the number of patients with postbaseline HRQoL data, particularly those with evaluable response, is relatively small and decreases in later cycles. As HRQoL was an exploratory end point in SADAL, results should be interpreted considering the study design and number of patients included in the analyses. In the responder analysis, the mixedeffects model assumed a linear relationship between time and responder status, and that patient trajectories would remain similar following discontinuation from the study. As patients were not randomized according to responder and nonresponder status, comparison of HRQoL change by responder status may be confounded by differences in unmeasured clinical characteristics which results in residual confounding. Due to the exploratory nature of the analysis and small patient numbers, the analysis was not powered to explore the relationship between HRQoL and disease-specific characteristics such as de novo or transformed DLBCL, genetic subtypes or prior number of therapies. Notably in SADAL, response to selinexor was maintained across patients with de novo or transformed DLBCL, GCB or non-GCB subtype, $>2$ prior systemic anti-DLBCL regimens and those who had previously received ASCT, with overall response rates ranging from 20.6 to 38.7\% [42]. While this work does not define new insights into clinical course of the disease, it contributes to an understanding of how selinexor treatment impacts HRQoL, which is an outcome relevant to patients, clinicians, health economists and other stakeholders. The current analysis identified that HRQoL outcomes with selinexor were consistent with the durable clinical response and delayed disease progression observed in patients with objective response. Additional analyses in larger patient populations and real-world settings are warranted to further validate these findings.

\section{Conclusion}

In summary, analyses showed that patients with RR DLBCL who responded to treatment with single-agent selinexor in the SADAL trial maintained higher HRQoL and health utilities whereas nonresponders experienced deterioration, which was clinically meaningful. Treatment responders had higher mean health state utility compared with patients with progressive disease and stable disease. This evidence complements the clinical benefits and manageable $\mathrm{AE}$ profile of oral single-agent selinexor, which provided durable and consistent responses in heavily pretreated patients with RR DLBCL. RR DLBCL-relevant health state utility values and disutilities derived in this study may be applicable to future health economic evaluations.

\footnotetext{
Author contributions

P Daniele, D Tomaras and G Tremblay were responsible for data analyses and data interpretation and participated in drafting and revising of the manuscript. All authors were responsible for study conception and design and participated in data interpretation and reviewed the manuscript. All authors participated in study design and data interpretation, and reviewed the manuscript.
}

Acknowledgments

The authors acknowledge and thank the patients who participated in the SADAL trial, their families, caregivers, and the study staff and healthcare providers at all SADAL trial sites. 
Financial \& competing interests disclosure

This work was funded by Karyopharm Therapeutics. P Daniele, D Tomaras and G Tremblay are employees of Purple Squirrel Economics, which received funding from Karyopharm Therapeutics to conduct the analysis. The authors have no other relevant affiliations or financial involvement with any organization or entity with a financial interest in or financial conflict with the subject matter or materials discussed in the manuscript apart from those disclosed.

Writing assistance was provided by N Vurgun and K Sandman, who are employees of Purple Squirrel Economics. The authors are fully responsible for all content and editorial decisions in this manuscript.

\section{Ethical conduct of research}

This study was performed in compliance with the ethical principles that originate from the Declaration of Helsinki and are consistent with International Council for Harmonisation guidelines on Good Clinical Practice and regulatory requirements as applicable. The study protocol was approved by the institutional review board or an independent ethics committee at each study center. Written informed consent in accordance with federal, local and institutional guidelines was obtained from all patients.

\section{Data sharing statement}

The authors certify that this manuscript reports secondary analysis of clinical trial data that was shared with them, and that the use of this shared data is in accordance with the terms agreed upon their receipt. The source of this data is NCT02227251 (Karyopharm Therapeutics).

\section{Open access}

This work is licensed under the Attribution-NonCommercial-NoDerivatives 4.0 Unported License. To view a copy of this license, visit http://creativecommons.org/licenses/by-nc-nd/4.0/

\section{Summary points}

- SADAL was a Phase Ilb, single-arm, open-label, multicenter trial of single-agent, oral selinexor in patients with relapsed/refractory diffuse large B-cell lymphoma (RR DLBCL) who were not eligible for transplantation and had 2-5 prior systemic therapies.

- This study examined the impact of selinexor on patient-reported health-related quality of life (HRQoL) as assessed by the Functional Assessment of Cancer Therapy - Lymphoma (FACT-Lym) and EuroQoL five-dimensions five-levels (EQ-5D-5L) in the SADAL trial.

- Multivariable mixed-effects models were used to estimate changes in baseline in FACT - General, the FACT-Lym Trial Outcome Index, the FACT-Lym total score and EQ-5D-5L index scores. In addition, health state utility values and disutilities of grade $\geq 3$ and serious adverse events (AEs) were estimated.

- The HRQoL and health utility longitudinal analyses included 101 and 89 patients, respectively, who had completed a baseline and $\geq 1$ postbaseline assessment and had complete baseline prognostic data.

- Patients with partial or complete response to selinexor maintained higher HRQoL than nonresponders across all cycles of treatment, with statistically significant and clinically meaningful changes beginning in cycle 3 for FACT General and FACT-Lym total score $(p \leq 0.05)$.

- Patients with objective response (i.e., treatment responders) had a significantly higher mean EQ-5D-5L index score than nonresponders by cycle 3, which was maintained through to cycle 7 (mean difference: 0.249 [95\% Cl: $0.095-0.402$ ]; $p<0.001$ ). Treatment responders experienced no clinically meaningful change in health utility from baseline at any cycle.

- Estimated mean health state utility values at the end of treatment were $0.669(95 \% \mathrm{Cl}: 0.619-0.719)$ for progressive disease, 0.721 ( $95 \% \mathrm{Cl}: 0.658-0.784)$ for stable disease, and 0.739 (95\% Cl: 0.689-0.790) for treatment response. The estimated difference in health state utilities between treatment response and progressive disease was statistically significant and clinically meaningful, as it exceeded the lower threshold of the minimal clinically meaningful differences (mean difference: $0.07 ; p=0.001$ ).

- Grade $\geq 3$ AEs and serious AEs with incidence $\geq 10 \%$ in SADAL were associated with significant decrements in EQ-5D-5L index scores (both $p=0.002$ ). However, disutilities associated with these AEs did not reach the lower threshold of the minimal clinically meaningful differences to be clinically meaningful.

- HRQoL maintenance in treatment responders suggests a favorable benefit-risk profile of selinexor, given its durable response, tolerability and safety profile in patients with RR DLBCL.

- RR DLBCL-relevant health state utility values and disutilities reported in this study may be applicable to future health economic evaluations. 


\section{References}

Papers of special note have been highlighted as: $\bullet$ of interest; $\bullet \bullet$ of considerable interest

1. Li S, Young KH, Medeiros LJ. Diffuse large B-cell lymphoma. Pathology 50(1), 74-87 (2018).

2. Chen B-J, Fend F, Campo E, Quintanilla-Martinez L. Aggressive B-cell lymphomas: from morphology to molecular pathogenesis. Ann. Lymphoma 3, 1-1 (2019).

3. Teras LR, DeSantis CE, Cerhan JR, Morton LM, Jemal A, Flowers CR. 2016 US lymphoid malignancy statistics by World Health Organization subtypes. CA Cancer J. Clin. 66(6), 443-459 (2016).

4. National Cancer Institute. SEER Cancer Stat Facts: NHL - diffuse large B-cell lymphoma (DLBCL). https://seer.cancer.gov/statfacts/html/dlbcl.html

5. National Comprehensive Cancer Network. NCCN clinical practice guidelines in oncology (NCCN guidelines ${ }^{\circledR}$ ) for B-Cell lymphomas V.4.2020 (2020). https://www.nccn.org/professionals/physician_gls/pdf/b-cell.pdf

6. Liu Y, Barta SK. Diffuse large B-cell lymphoma: 2019 update on diagnosis, risk stratification, and treatment. Am. J. Hematol. 94(5), 604-616 (2019).

7. Sarkozy C, Sehn LH. Management of relapsed/refractory DLBCL. Best Pract. Res. Clin. Haematol. 31(3), 209-216 (2018).

8. Skrabek P, Assouline S, Christofides A et al. Emerging therapies for the treatment of relapsed or refractory diffuse large B cell lymphoma. Curr. Oncol. 26(4), 253-265 (2019).

9. Coiffier B, Lepage E, Brière J et al. CHOP chemotherapy plus rituximab compared with $\mathrm{CHOP}$ alone in elderly patients with diffuse large-B-cell lymphoma. N. Engl. J. Med. 346(4), 235-242 (2002).

10. Coiffier B, Thieblemont C, van den Neste E et al. Long-term outcome of patients in the LNH-98.5 trial, the first randomized study comparing rituximab-CHOP to standard CHOP chemotherapy in DLBCL patients: a study by the Groupe d'Etudes des Lymphomes de l'Adulte. Blood 116(12), 2040-2045 (2010).

11. Crump M, Neelapu SS, Farooq U et al. Outcomes in refractory diffuse large B-cell lymphoma: results from the international SCHOLAR-1 study. Blood 130(16), 1800-1808 (2017).

12. Crump M, Kuruvilla J, Couban S et al. Randomized comparison of gemcitabine, dexamethasone, and cisplatin versus dexamethasone, cytarabine, and cisplatin chemotherapy before autologous stem-cell transplantation for relapsed and refractory aggressive lymphomas: NCIC-CTG LY.12. J. Clin. Oncol. 32(31), 3490-3496 (2014).

13. Nagle SJ, Woo K, Schuster SJ et al. Outcomes of patients with relapsed/refractory diffuse large B-cell lymphoma with progression of lymphoma after autologous stem cell transplantation in the rituximab era. Am. J. Hematol. 88(10), 890-894 (2013).

14. Gisselbrecht C, Glass B, Mounier N et al. Salvage regimens with autologous transplantation for relapsed large B-cell lymphoma in the rituximab era. J. Clin. Oncol. 28(27), 4184-4190 (2010).

15. Thompson CA, Ghesquieres H, Maurer MJ et al. Utility of routine post-therapy surveillance imaging in diffuse large B-cell lymphoma. J. Clin. Oncol. 32(31), 3506-3512 (2014).

16. Ahmed MA, Chihara D, Vargas N et al. Outcome of relapsed/refractory diffuse large B-cell lymphoma after second salvage therapy: MD Anderson experience. Hematol. Oncol. 33, 279 (2015).

17. Maurer MJ, Ghesquières H, Jais J-P et al. Event-free survival at 24 months is a robust end point for disease-related outcome in diffuse large B-cell lymphoma treated with immunochemotherapy. J. Clin. Oncol. 32(10), 1066-1073 (2014).

18. $\mathrm{Xpovio}^{\circledR}$, prescribing information. Karyopharm Therapeutics, Inc., MA, USA, (2020).

19. Yoshimura M, Ishizawa J, Ruvolo V et al. Induction of $\mathrm{p} 53$-mediated transcription and apoptosis by exportin-1 (XPO1) inhibition in mantle cell lymphoma. Cancer Sci. 105(7), 795-801 (2014).

20. Gravina GL, Senapedis W, McCauley D, Baloglu E, Shacham S, Festuccia C. Nucleo-cytoplasmic transport as a therapeutic target of cancer. J. Hematol. Oncol. 7(1), 85 (2014).

21. Wang AY, Liu H. The past, present, and future of CRM1/XPO1 inhibitors. Stem Cell Investig. 6, 6 (2019).

22. Clinical trial registration: NCT02227251. A Phase IIb open-label study of selinexor (KPT-330) in patients with relapsed/refractory diffuse large B-cell lymphoma (DLBCL). https://clinicaltrials.gov/ct2/show/NCT02227251

23. Kalakonda N, Maerevoet M, Cavallo F et al. Selinexor in patients with relapsed or refractory diffuse large B-cell lymphoma (SADAL): a single-arm, multinational, multicentre, open-label, Phase II trial. Lancet Haematol. 7(7), e511-e522 (2020).

-. The manuscript reporting SADAL trial primary results of clinical outcomes and safety with single-agent selinexor in patients with relapsed/refractory diffuse large B-cell lymphoma.

24. Oerlemans S, Issa DE, van den Broek EC et al. Health-related quality of life and persistent symptoms in relation to (R-)CHOP14, (R-)CHOP21, and other therapies among patients with diffuse large B-cell lymphoma: results of the population-based PHAROS-registry. Ann. Hematol. 93(10), 1705-1715 (2014).

25. Price M, Ravelo A, Sae-Hau M et al. Patient-reported disease burden in chronic lymphocytic leukemia, diffuse large B-cell lymphoma, and follicular lymphoma: results from a national patient advocacy survey. J. Clin. Oncol. 37(Suppl. 15), e18198-e18198 (2019). 
26. Arboe B, Goerloev JS, Olsen MH et al. Social outcomes after high dose chemotherapy with autologous stem cell transplant. Blood 128(22), 1193-1193 (2016).

27. Wang H, Manca A, Crouch S et al. PCN351 - health-state utility values in diffuse large B-cell lymphoma. Value Health 21, S74 (2018).

28. Kelly JL, Pandya C, Friedberg JW, Mohile SG. Health-related quality of life in older patients following diffuse large B-cell lymphoma (DLBCL) diagnosis. Blood 120(21), 4287-4287 (2012).

29. Efficace F, Gaidano G, Lo-Coco F. Patient-reported outcomes in hematology: is it time to focus more on them in clinical trials and hematology practice? Blood 130(7), 859-866 (2017).

30. Au H-J, Ringash J, Brundage M, Palmer M, Richardson H, Meyer RM. Added value of health-related quality of life measurement in cancer clinical trials: the experience of the NCIC CTG. Expert Rev. Pharmacoecon. Outcomes Res. 10(2), 119-128 (2010).

31. US Department of Health and Human Services, USFDA. Guidance for industry: patient-reported outcome measures: use in medical product development to support labeling claims. Health. Qual. Life Outcome 4(79), doi: 10.1186/1477-7525-4-79 (2006).

32. European Medicine Agency. The Use of Patient-Reported Outcome (PRO) Measures in Oncology Studies. Appendix 2 to the Guideline on the Evaluation of Anticancer Medicinal Products in Man (2016).

https://www.ema.europa.eu/en/documents/other/appendix-2-guideline-evaluation-anticancer-medicinal-products-man_en.pdf

33. Kluetz PG, Slagle A, Papadopoulos EJ et al. Focusing on core patient-reported outcomes in cancer clinical trials: symptomatic adverse events, physical function, and disease-related symptoms. Clin. Cancer Res. 22(7), 1553-1558 (2016).

34. Basch E. Toward patient-centered drug development in oncology. N. Engl. J. Med. 369(5), 397-400 (2013).

35. Basch E, Geoghegan C, Coons SJ et al. Patient-reported outcomes in cancer drug development and US regulatory review: perspectives from industry, the Food and Drug Administration, and the patient. JAMA Oncol. 1(3), 375 (2015).

36. Cella D, Webster K, Cashy J et al. Development of a measure of health-related quality of life for non-Hodgkin's lymphoma clinical research: the functional assessment of cancer therapy - lymphoma (FACT-Lym). Blood 106(11), 750-750 (2005).

- A review article reporting the range of minimal important differences for the Functional Assessment of Cancer Therapy (FACT) - General (FACT-G) total score.

37. Hlubocky FJ, Webster K, Cashy J, Beaumont J, Cella D. The development and validation of a measure of health-related quality of life for non-Hodgkin's lymphoma: the functional assessment of cancer therapy - lymphoma (FACT-Lym). Lymphoma 2013, 1-9 (2013).

38. Wiebe S, Guyatt G, Weaver B, Matijevic S, Sidwell C. Comparative responsiveness of generic and specific quality-of-life instruments. J. Clin. Epidemiol. 56(1), 52-60 (2003).

39. Pickard AS, Wilke CT, Lin H-W, Lloyd A. Health utilities using the EQ-5D in studies of cancer. Pharmacoeconomics 25(5), 365-384 (2007).

40. van Hout B, Janssen MF, Feng Y-S et al. Interim scoring for the EQ-5D-5L: mapping the EQ-5D-5L to EQ-5D-3L value sets. Value Health 15(5), 708-715 (2012).

41. Herdman M, Gudex C, Lloyd A et al. Development and preliminary testing of the new five-level version of EQ-5D (EQ-5D-5L). Qual. Life Res. 20(10), 1727-1736 (2011).

42. Kalakonda N, Maerevoet M, Cavello F et al. Selinexor in patients with relapsed or refractory diffuse large B-cell lymphoma (SADAL): a single-arm, multinational, multicentre, open-label, Phase II trial. Lancet Haematol. 7(7), e511-e522 (2020).

43. Dreyling M, Jurczak W, Jerkeman $\mathrm{M}$ et al. Ibrutinib versus temsirolimus in patients with relapsed or refractory mantle-cell lymphoma: an international, randomised, open-label, Phase 3 study. Lancet 387(10020), 770-778 (2016).

44. Hess G, Rule S, Jurczak W et al. Health-related quality of life data from a Phase III, international, randomized, open-label, multicenter study in patients with previously treated mantle cell lymphoma treated with ibrutinib versus temsirolimus. Leuk. Lymphoma 58(12), 2824-2832 (2017).

45. Cheson BD, Trask PC, Gribben JG et al. Health-related quality of life and symptoms in patients with rituximab-refractory indolent non-Hodgkin lymphoma treated in the Phase III GADOLIN study with obinutuzumab plus bendamustine versus bendamustine alone. Ann. Hematol. 96(2), 253-259 (2017).

46. Carter GC, Liepa AM, Zimmermann AH, Morschhauser F. Validation of the functional assessment of cancer therapy-lymphoma (FACT-LYM) in patients with relapsed/refractory mantle cell lymphoma. Blood 112(11), 2376-2376 (2008).

- A previous validation of the FACT - Lymphoma (FACT-Lym) assessment that determined minimally important differences in patients with non-Hodgkin lymphoma for the FACT-Lym total score and FACT-Lym Trial Outcome Index.

47. Maziarz RT, Waller EK, Jaeger U et al. Patient-reported long-term quality of life after tisagenlecleucel in relapsed/refractory diffuse large B-cell lymphoma. Blood Adv. 4(4), 629-637 (2020).

- Analyses of long-term health-related quality of life outcomes measured with the FACT-Lym and Short-Form 36 in adult patients with relapsed/refractory diffuse large B-cell lymphoma treated with a single infusion of tisagenlecleucel in the JULIET Phase II trial.

48. van Reenen M, Janssen B, Stolk E et al. EuroQol research foundation. EQ-5D-5L user guide (version 3).

(2019). https://euroqol.org/eq-5d-instruments/ 
49. Cheson BD, Fisher RI, Barrington SF et al. Recommendations for initial evaluation, staging, and response assessment of Hodgkin and non-Hodgkin lymphoma: the Lugano classification. J. Clin. Oncol. 32(27), 3059-3067 (2014).

50. Jaeschke R, Singer J, Guyatt GH. Measurement of health status. Control. Clin. Trials 10(4), 407-415 (1989).

51. Jayadevappa R, Cook R, Chhatre S. Minimal important difference to infer changes in health-related quality of life: a systematic review. $J$. Clin. Epidemiol. 89, 188-198 (2017).

52. Pickard AS, Neary MP, Cella D. Estimation of minimally important differences in EQ-5D utility and VAS scores in cancer. Health Qual. Life Outcomes 5(1), 70 (2007).

- A validation study of the EuroQoL five-dimensions five-levels that recommends a threshold of 0.06 for identifying minimal clinically important differences in US-index scores in patients with cancer.

53. Kvam AK, Fayers PM, Wisloff F. Responsiveness and minimal important score differences in quality-of-life questionnaires: a comparison of the EORTC QLQ-C30 cancer-specific questionnaire to the generic utility questionnaires EQ-5D and 15D in patients with multiple myeloma: responsiveness of questionnaires in MM. Eur. J. Haematol. 87(4), 330-337 (2011).

- An analysis that suggested a threshold of 0.08-0.1 for identifying clinically meaningful differences in EuroQoL five-dimensions five-levels index scores among patients with multiple myeloma.

54. Patrick DL, Powers A, Parisi M et al. Impact of lisocabtagene maraleucel (liso-cel) treatment on health-related quality of life and health utility in patients (pts) with relapsed/refractory (R/R) aggressive B cell non-Hodgkin lymphoma (NHL): transcend NHL 001. Blood 134(Suppl. 1), 66-66 (2019).

55. Patrick DL, Chung KC, Kim Y, Garcia J, Dehner C, Maloney DG. TRANSCEND NHL 001: health-related quality of life (HRQL) and symptom (Sx) impact in patients (pts) with relapsed/refractory diffuse large B-cell lymphoma (R/R DLBCL) receiving lisocabtagene maraleucel (Liso-cel; JCAR017). J. Clin. Oncol. 37(Suppl. 15), e19052-e19052 (2019).

56. Thuresson P-O, Gentile B, Ramies D, Launonen A, Morschhauser F. Quality of life (QoL) in patients with relapsed/refractory non-Hodgkin lymphoma (NHL) treated with polatuzumab vedotin plus rituximab in the ROMULUS study. Blood 134(Suppl. 1), 4767-4767 (2019).

57. Straus DJ, Hamlin PA, Matasar MJ et al. Phase I/II trial of vorinostat with rituximab, cyclophosphamide, etoposide and prednisone as palliative treatment for elderly patients with relapsed or refractory diffuse large B-cell lymphoma not eligible for autologous stem cell transplantation. Br. J. Haematol. 168(5), 663-670 (2015).

58. Morschhauser F, Flinn IW, Advani R et al. Polatuzumab vedotin or pinatuzumab vedotin plus rituximab in patients with relapsed or refractory non-Hodgkin lymphoma: final results from a Phase II randomised study (ROMULUS). Lancet Haematol. 6(5), e254-e265 (2019).

59. NICE. Tisagenlecleucel for treating relapsed or refractory diffuse large B-cell lymphoma after 2 or more systemic therapies: technology appraisal guidance [TA567]. (2019). https://www.nice.org.uk/guidance/ta567

60. Norwegian Medicines Agency. Single technology assessment - tisagenlecleucel (kymriah) for the treatment of second or later relapsed/refractory diffuse large B cell Lymphoma (DLBCL). (2019). https://legemiddelverket.no/Documents/Offentlig\%20finansiering\%20og\%20pris/Metodevurderinger/K/Kymriah_DLBCL_2019.pdf

61. Patrick D, Matza L, Kim Y, Garcia J, Dehner C, Chung KC. PCN262 preference-weighted health status in patients with relapsed/refractory diffuse large B-cell lymphom A (R/R DLBCL) treated with lisocabtagene maraleuCEL (LISO-CEL; JCAR017) in the ongoing, multicenter, Phase I transcend NHL 001 trial. Value Health 22, S106 (2019).

62. NICE. Axicabtagene ciloleucel for treating diffuse large B-cell lymphoma and primary mediastinal large B-cell lymphoma after 2 or more systemic therapies: technology appraisal guidance [TA559]. (2019). https://www.nice.org.uk/guidance/ta559

63. Neelapu SS, Locke FL, Bartlett NL et al. Axicabtagene ciloleucel CAR T-cell therapy in refractory large B-cell lymphoma. N. Engl. J. Med. 377(26), 2531-2544 (2017). 